\title{
Environment and Behavior Influence the Complexity of Evolved Neural Networks
}

\author{
Anil K. Seth, Gerald M. Edelman \\ The Neurosciences Institute, San Diego
}

\begin{abstract}
How does environmental structure influence the dynamics of adaptive behavior and its underlying mechanisms? By analyzing the neural controller of a simulated head/eye system, we show that a specific measure-"neural complexity"-can be selectively sensitive to neural dynamics underlying rich adaptive behavior. Evolutionary algorithms were used to generate neural network controllers able to support target fixation in environmental and phenotypic conditions of qualitatively different complexity. Networks that evolved in rich conditions showed higher behavioral flexibility and robustness, and higher neural complexity, than networks that evolved in simple conditions. The magnitude of neural complexity, which reflects a balance between dynamical integration and dynamical segregation, depended on properties of both the environment and the head/eye phenotype. These results show that neurally complex dynamics can accompany adaptive behavior in rich environmental and phenotypic conditions; they are consistent with the proposal that neural complexity may represent a common property of the functional organization of adaptive neural systems.
\end{abstract}

Keywords environment · behavior $\cdot$ mechanism · neural complexity · information theory · graph theory

\section{Introduction}

Understanding the interactions among environmental complexity, behavioral complexity, and organism complexity is a central problem in the study of adaptive behavior (Seth, 2000). From one point of view, organism complexity may be best understood in terms of adaptation to complexity in the environment (see Young, 1970; Godfrey-Smith, 1996). Alternatively, organism complexity is sometimes seen as the result of "self-organizing" properties of biological systems (Thompson, 1917; Kauffman, 1993). Of course, these positions are not mutually exclusive, nor is organism or mechanism complexity necessarily a prerequisite for behavioral complexity. Both Simon (1988) and Braitenberg (1984) have pointed out that surprisingly

Correspondence to: A. K. Seth, The Neurosciences Institute, 10640 John Jay Hopkins Drive, San Diego, CA 92121, USA. E-mail: seth@nsi.edu Tel.: +1-858-626-2000, Fax: +1-858-626-2099. complex behaviors can be supported by simple mechanisms, given a sufficiently rich environment.

Evolutionary simulation modeling provides a powerful means for exploring these issues in detail. Comparative analyses can be conducted among artificial "organisms", or mechanisms, evolved in differently structured environments. Previous work using this technique has shown a qualitative correspondence between environmental complexity and evolved behavioral/mechanistic complexity in a variety of contexts. For example, in the evolution of strategies to play the iterated prisoner's dilemma, occasional errors in the perception or execution of a strategy have been found to promote the evolution of strategies that make use of comparatively long memories (Lindgren, 1991; Seth, 1997). A second example is provided by the evo-

Copyright (C) 2004 International Society for Adaptive Behavior (2004), Vol 12(1): 5-20.

[1059-7123(200403) 12:1; 5-20; 044126] 
lution of neural network controllers for mobile robots engaged in a homing navigation task, in which unpredictable and highly variable environments favored the evolution of controllers capable of multimodal sensor integration (Seth, 1998; Nolfi \& Floreano, 2000). A number of other studies have found that evolved behaviors and mechanisms can be related to environmental complexity (Menczer \& Belew, 1996; Todd \& Yanco, 1996; Fletcher, Zwick, \& Bedau, 1998).

The present paper extends this previous work by introducing a quantitative methodology for relating behavioral, mechanistic, and environmental complexity. Based on recent advances in theoretical neuroscience (Tononi, Sporns, \& Edelman, 1994; Sporns, Tononi, \& Edelman, 2000), this methodology combines evolutionary simulation modeling (Nolfi \& Floreano, 2000; Di Paolo, Noble, \& Bullock, 2000) with aspects of graph theory (Bollobás, 1985; Albert \& Barábasi, 2002; Newman, 2003) and statistical information theory (Shannon \& Weaver, 1949). As "mechanisms" we consider neural networks consisting of $N$ $=32$ nodes and $K=256$ weighted, directed connections. Evolutionary algorithms are used to specify the connectivity and weight distribution of these networks so that, when employed as control mechanisms, they give rise to adaptive behavior. Here, such behavior consists of target fixation in a simulated head/eye system (the phenotype), a context that allows for environments and phenotypes of (qualitatively) tunable complexity. Networks that evolved in different environmental/phenotypic conditions are then analyzed in terms of their behavioral properties, their structural properties (using methods from graph theory), and their dynamical properties (using methods based on statistical information theory).

Our methods of dynamical analysis have the important advantage of being equally applicable to both weighted and unweighted (i.e., binary) networks; structural analysis, by contrast, is usually best suited to binary networks. We also make a novel distinction between "intrinsic" and "interactive" network dynamics. The measurement of intrinsic dynamics follows previous work (Tononi et al., 1994; Sporns et al., 2000) by assuming: (1) every node in the network has an independent Gaussian noise input; (2) nodes implement linear input-output functions. Under these assumptions, dynamical properties can be derived analytically from network connectivity and are independent of behavioral context. An alternative approach, developed in this paper, is to record the activity of each node in a network while the network is actively transforming input signals into output signals. We define the resulting dynamics as "interactive", since they are relative to a particular behavior, phenotype, and environment. One important property of interactive dynamics is that they enable comparison of dynamics generated by the same network in different behavioral regimes or in different environments.

Using the methods described earlier, we examine how intrinsic and interactive measures of neural complexity relate to behavior and environment, where neural complexity is a measure of network dynamics that captures quantitatively the interplay between dynamical integration and dynamical segregation in a neural system (Tononi et al., 1994). Specifically, we test the hypothesis that neurally complex dynamics accompany adaptation to rich sensory environments and motor demands.

We find that networks that evolved to support target fixation in rich environmental and phenotypic conditions show greater behavioral robustness and flexibility than those that evolved in comparatively simple conditions, even when compared in novel environments. We also find that this robustness is accompanied by higher neural complexity, whether measured intrinsically or interactively; the association is particularly clear for interactive neural complexity. The magnitude of neural complexity is found to depend on properties of both the environment and the phenotype. No differences are apparent when these networks are analyzed in terms of standard structural criteria, most likely because such criteria are not sensitive to differences in connection strengths. Overall, our results show that neural complexity can be selectively sensitive to neural dynamics underlying adaptive behavior in rich environmental and phenotypic conditions, and they are consistent with the proposal that neural complexity may reflect a common property of the functional organization of adaptive neural systems.

The paper is organized as follows. Section 2 describes the structure of the model. The results of experiments carried out with the model are described in Section 3, which is divided into three parts: a behavioral analysis, a structural analysis of evolved mechanisms, and a dynamical analysis of evolved 
mechanisms. The implications of these analyses are discussed in Section 4.

\section{Methods}

\subsection{Model Outline}

We analyzed the influence of environment on behavior and its underlying mechanisms using a simulation model of target fixation requiring coordination of "head" and "eye" movements. This section describes basic properties of the model; specifics are provided in Appendix A. The simulated environment is a simple planar area, $100 \times 100$ arbitrary units $u$, within which a target $(\mathrm{T})$ can appear. The simulated head/eye system (the phenotype) is represented by the projection onto the plane of a head direction $(\mathrm{H})$ and a gaze direction $(\mathrm{G})$, which is the combination of the head direction $(\mathrm{H})$ and an eye direction $(\mathrm{E})$ relative to the head (Figure 1).

The velocities of $\mathrm{H}$ and $\mathrm{E}$ in the $x, y$ plane, and thus the position of $\mathrm{G}$, are controlled by a neural network with $N=32$ nodes and $K=256$ weighted, directed, and initially randomly allocated connections. Certain nodes in the network are specified as sensory inputs and others as motor outputs (see Section 2.3), and the remainder are "interneurons" mediating the transformation of input signals into output signals.

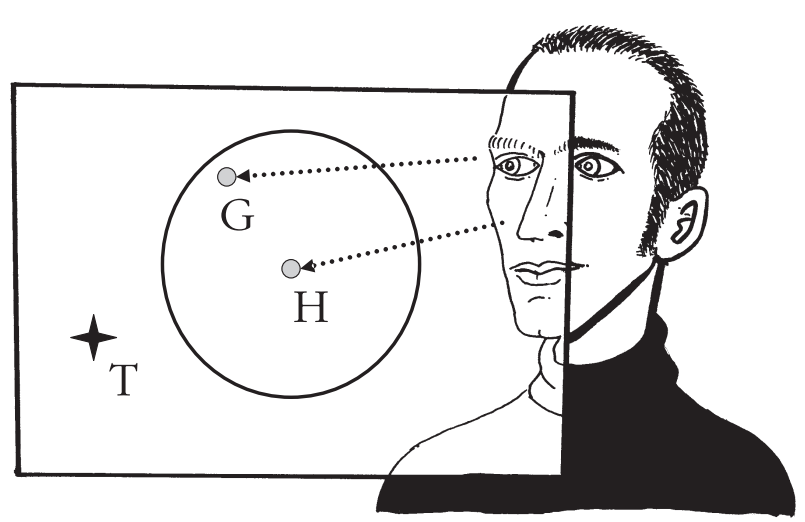

Figure 1 Simulation model of target fixation, depicting projections onto a plane $(100 u \times 100 u$, where $u$ denotes an arbitrary spatial unit) of head direction $\mathrm{H}$ and gaze direction $\mathrm{G}$. Also shown are target position $\mathrm{T}$, and maximum offset of $\mathrm{G}$ from $\mathrm{H}$ (circle, radius $35 \mathrm{u}$ ). The position of $\mathrm{G}$ is calculated as a vector sum of $\mathrm{H}$ and an eye direction (E, not shown).
Evolutionary algorithms are used to specify the connectivity and weight distribution of networks (see section 2.4), so that they give rise to adaptive behavior, which in this case consists of maximizing the time for which $\mathrm{G}$ and $\mathrm{T}$ are aligned (i.e., keeping the target fixated), while simultaneously minimizing the offset between $\mathrm{H}$ and $\mathrm{G}$ (i.e., keeping the head and eye aligned). A constraint on the system is that $G$ must remain within $35 u$ of $\mathrm{H}$, as indicated by the circle in Figure 1. Note that this model is not intended to faithfully represent biological mechanisms of eye movements or target fixation (Shibata, Vijayakumar, Conradt, \& Schaal, 2001). Rather, it is intended as a tool for investigating interactions between environment, behavior, and mechanism in a tunable and analytically tractable fashion.

\subsection{Environment/Phenotype Conditions}

We specify four experimental conditions that are distinguished by properties of the target and the head/eye phenotype. The phenotype is defined by seven parameters: $V_{\max H}$ and $V_{\max }$ specify the maximum velocities of $\mathrm{H}$ and $\mathrm{E}$; motor gain parameters $A_{H}$ and $A_{E}$ specify scaling factors relating motor node output to $\mathrm{H}$ and $\mathrm{E}$ velocity; momentum parameters $m_{H}$ and $m_{E}$ specify the inertial resistance of $\mathrm{H}$ and $\mathrm{E}$, and lag specifies a time-lag between head motor node output and head movement. These parameters constrain the movements of $\mathrm{H}, \mathrm{E}$, and $\mathrm{G}$ as described in Appendix A (which also describes how $\mathrm{T}$ is updated).

The values taken by the parameters in the four conditions are shown in Table 1. In condition $E_{S}$ (for "simple") both the head and eye have the same maximum velocity, identical motor gains, and zero momentum; the target is stationary. Condition $E_{T}$ (for "tracking") presents a more complex environment in which the target may occasionally jump to a different random location and/or drift at a slow speed in a random direction; the phenotype is the same in condition $E_{S}$. Condition $E_{H}$ (for "head") keeps the simple environment of condition $E_{S}$, but introduces a more complex phenotype in which $\mathrm{E}$ can move twice as fast as $\mathrm{H}$, has a higher motor gain, a much lower momentum, and in which there is a non-zero time-lag. Finally, condition $E_{C}$ (for "complex") combines the properties of both conditions $E_{T}$ and $E_{H}$ and is therefore the richest of the four. 
Table 1 Phenotype parameters in the four conditions $\left(E_{S}, E_{T}, E_{H}\right.$, and $\left.E_{C}\right)$.

\begin{tabular}{llllllll}
\hline Condition & $V_{\operatorname{maxH}}$ & $V_{\max E}$ & $A_{H}$ & $A_{E}$ & $m_{H}$ & $m_{E}$ & lag \\
\hline$E_{S}$ & 7.0 & 7.0 & 1.0 & 1.0 & 0.0 & 0.0 & 0 \\
$E_{T}$ & 7.0 & 7.0 & 1.0 & 1.0 & 0.0 & 0.0 & 0 \\
$E_{H}$ & 5.0 & 10.0 & 0.33 & 1.0 & 0.75 & 0.05 & 10 \\
$E_{C}$ & 5.0 & 10.0 & 0.33 & 1.0 & 0.75 & 0.05 & 10 \\
\hline
\end{tabular}

$V_{\max H}, V_{\max E}:$ maximum velocity of head and eye; $A_{H}, A_{E}$ : motor gain of head and eye; $m_{H}, m_{E}:$ momentum of head and eye; lag: the time-lag between head motor node output and head movement.

\subsection{Network Implementation}

Each behavioral trial begins with the head and eye aligned and pointing to the center of the plane. The target position is initialized at a randomly selected location within $20 u$ of this point. All trials last for 600 time-steps, each of which involves updating the state of the network controller $(\mathrm{X})$ as well as the positions of $\mathrm{E}, \mathrm{G}, \mathrm{H}$, and, in conditions $E_{T}$ and $E_{C}$ only, T. The network is updated using, for all nodes $j$,

$$
\begin{aligned}
s_{j} & =f\left(s_{i n}(j, t)+\sum_{i=1}^{K} \mathbf{C}_{\mathbf{i j}}(X) s_{i}(t-1)\right) \\
s_{j}(0) & =0.0
\end{aligned}
$$

where $s_{j}(t)$ is the output of node $j$ at time $t, s_{i n}(j, t)$ denotes the sensory input to node $j$ at time $t$ (if any), $f$ is a sigmoid function with input range \pm 10.0 and output range \pm 1.0 , and $\mathbf{C}_{\mathrm{ij}}(\mathrm{X})$ is a connection matrix holding the strengths of connections (if any) between nodes $i$ and $j$.

Six nodes are specified as "sensory" inputs, two responding to the $x, y$ displacement of $\mathrm{G}$ from $\mathrm{T}$ (with a maximum range of $\pm 50.0 u$ ), and four delivering "proprioceptive" information, two of which reflect the displacement of $\mathrm{H}$ from the center of the plane (range $\pm 50.0 \mathrm{u}$ ), and two of which reflect the displacement of $\mathrm{G}$ from $\mathrm{H}$ (range $\pm 35.0 u$ ). In all cases, input values are linearly scaled to the range \pm 1.0 . Four nodes are specified as output nodes, two influencing $\mathrm{H}$ velocity in the $x, y$ plane, and two influencing the velocity of $\mathrm{E}$. Appendix A describes how the activities of these nodes, along with the values in Table 1 , are used to update the positions of $\mathrm{E}, \mathrm{G}$, and $\mathrm{H}$.

\subsection{Network Structure}

Evolutionary algorithms (EA) were used to specify the connectivity and weight distribution of networks so that they supported target fixation behavior. We ran 40 separate EA: 10 replications of the selection process for each of the four environment/phenotype conditions. Each EA evolved a population of 64 networks over 2000 generations, with each network initialized (generation 0 ) by randomly allocating $K$ connections (each connection strength randomly in the range \pm 1.0 ), subject to the constraint that each node had eight incoming connections. Each generation involved evaluating the fitness of each network, as described later, and then using stochastic rankbased selection to replace low fitness networks with mutated versions of high-fitness networks. Each mutation of a network involved randomly rewiring one connection (preserving in-degree and full connectivity) and also modifying the strength of each connection (probability 0.05 per connection) in the range \pm 0.1 .

The fitness of a network was calculated as the mean of four separate behavioral trials, with the fitness of each trial $(\phi)$ given by

$$
\phi=\frac{t_{f}}{t_{\text {tot }}}+\frac{1}{4}\left(1.0-\frac{\bar{d}}{35}\right)
$$

where $t_{f}$ denotes the number of time-steps for which the target was fixated (within a tolerance of $3 u$ ), $t_{\text {tot }}$ the total number of time-steps in the trial (600), and $\bar{d}$ the mean offset between $\mathrm{H}$ and $\mathrm{G}$ for the trial. ${ }^{1}$ 
Table 2 Summary of target fixation performance.

\begin{tabular}{lll}
\hline Network type & Fixation $(\%)$ & $|\mathrm{G}-\mathrm{H}|(u)$ \\
\hline S-network & $98.81(0.01)$ & $0.28(0.07)$ \\
T-network & $92.43(0.51)$ & $0.92(0.08)$ \\
H-network & $96.15(0.79)$ & $1.94(0.19)$ \\
C-network & $84.76(1.23)$ & $15.41(0.50)$ \\
\hline
\end{tabular}

The mean percentage of each behavioral trial for which T was fixated (within a tolerance of $3 u$ ), and mean displacement of $\mathrm{G}$ from $\mathrm{H}$ for each network type are shown. Mean values (with their standard errors in parentheses) were calculated from ten behavioral trials of each network type in the corresponding environment/phenotype condition.

\section{Results}

The fittest member from each EA was analyzed in terms of behavior, structure, and dynamics. For convenience, networks which evolved in condition $E_{S}$ will be referred to as S-networks, with the same nomenclature for conditions $E_{C}$ (C-networks), $E_{T}$ (T-networks) and $E_{H}$ (H-networks).

\subsection{Behavioral Analysis}

The target fixation behavior of evolved networks was assessed by measuring the percentage of each behavioral trial for which the target was fixated. Table 2 shows that average fixation performance in each condition was very high for S-networks, T-networks, and H-networks, and slightly lower for C-networks.

Figure 2 shows representative examples of successful fixation behavior for S-networks and C-networks in the corresponding conditions $\left(E_{S}\right.$ and $E_{C}$ respectively). Inspection of the trajectories of the head and the eye (Figure 2a), and of the elaborate patterns of offsets between T, H, and G (Figure 2b), indicates that the behavioral dynamics for C-networks were qualitatively more complex than for S-networks. A non-trivial coordination of head and eye is needed in $E_{C}$ conditions in order to maintain consistent fixation of the target. The achievement of successful fixation by both networks types is reflected by a consistent zero offset between $\mathrm{T}$ and $\mathrm{G}$ (Figure 2b; second row in each panel). Table 2 affirms that the average displacements between $\mathrm{H}$ and $\mathrm{G}$ were considerably larger for C-networks than for all other network types, while target fixation was achieved reliably in all cases.
To compare better the behavioral properties of Snetworks and C-networks, they were reevaluated in a novel condition involving unexpected perturbations. As in condition $E_{S}$, the environment was initialized with a stationary target T. An evolved S-network or Cnetwork was then introduced and allowed to fixate. After 100 time-steps, a head velocity $\left(V_{h x}, V_{h y}\right)$ of between 1.0 and 3.0 ( $u$ per time-step) was induced for 20 time-steps in a random direction. Importantly, neither network type had been selected to respond adaptively to this perturbation. Nevertheless, Figure 3 illustrates that $\mathrm{C}$-networks were generally able to maintain fixation, whereas S-networks were not. These results show that network optimization in a rich environmental/phenotypic context can facilitate the emergence of robust behavior.

\subsection{Structural Analysis}

Figure 4 illustrates representative examples of the types of networks that the evolutionary algorithm produced, for a C-network (top) and an S-network (bottom). The networks are shown in graph form $G(\mathrm{X})$, together with the corresponding $\mathbf{C}_{\mathrm{ij}}(\mathrm{X})$ matrices. The vertices of each graph have been reordered so that vertices that shared many of the same connections are placed next to each other, hence the small amount of clustering around the diagonal of the $\mathbf{C}_{\mathrm{ij}}(\mathrm{X})$ matrices.

To assay reliable structural differences between experimental conditions, we used graph theory to quantify structural aspects of evolved networks (Bollobás, 1985; Albert \& Barábasi, 2002; Newman, 2003). To enable this approach, connectivity matrices were transformed into binary adjacency matrices 


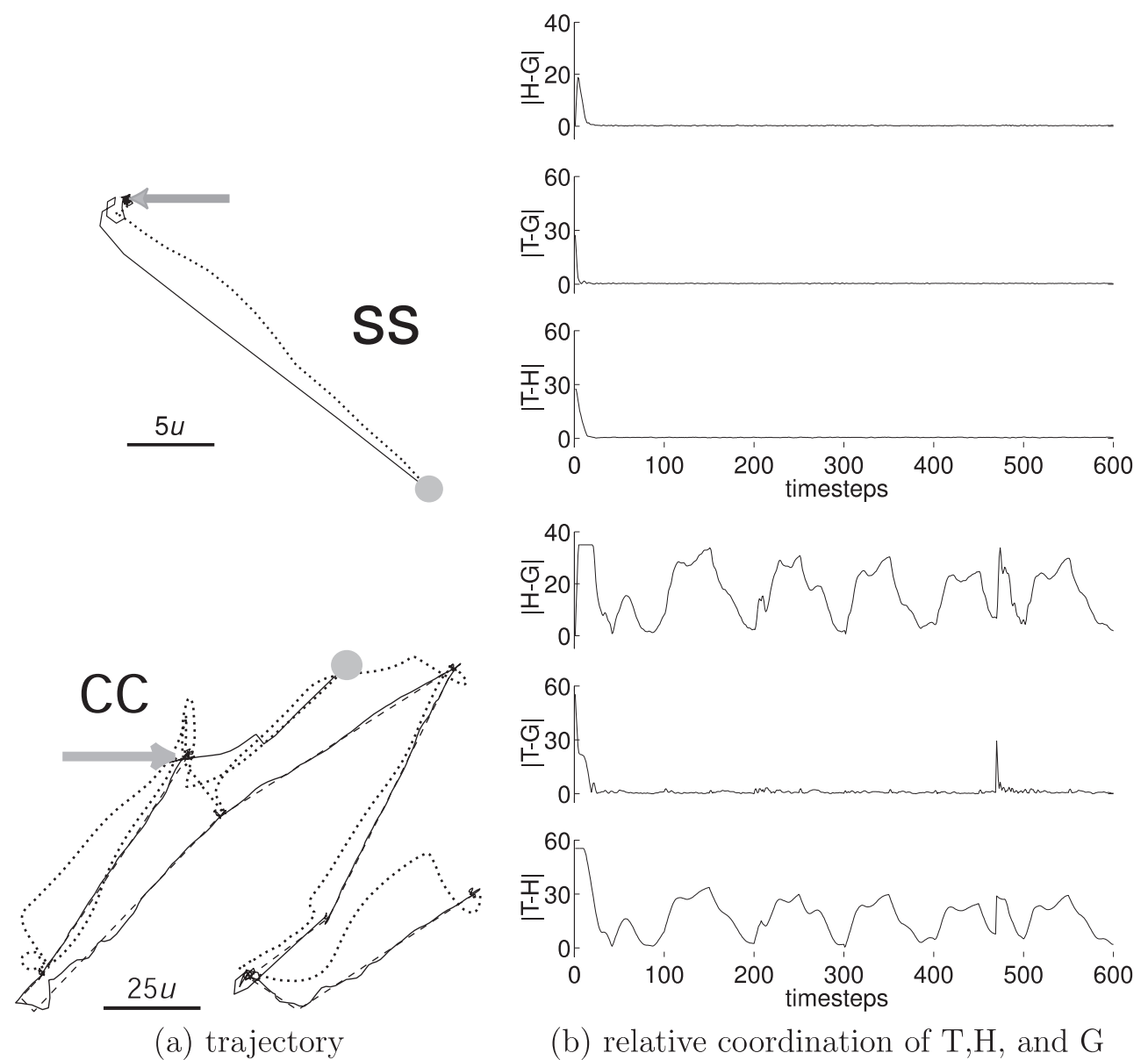

Figure 2 Typical successful fixation behavior for an S-network in condition $E_{S}$ (top) and a C-network in condition $E_{C}$ (bottom). (a) Trajectories of $\mathrm{G}$ (solid line), $\mathrm{H}$ (dotted line) and $\mathrm{T}$ (dashed line, $E_{C}$ only), gray circle indicates initial positions of $\mathrm{G}$ and $\mathrm{H}$, gray arrows indicate initial positions of $T$. (b) Representations of these trajectories: $H-G$, displacement of $\mathrm{H}$ from $\mathrm{G}$; $\mathrm{T}-\mathrm{G}$, offset of $\mathrm{T}$ from $\mathrm{G}$; $\mathrm{T}-\mathrm{H}$, offset of $\mathrm{T}$ from $\mathrm{H}$. Units along the $y$-axes of these plots are $u$. Behavioral dynamics were qualitatively more mplex for the $\mathrm{C}$-network than for the S-network.

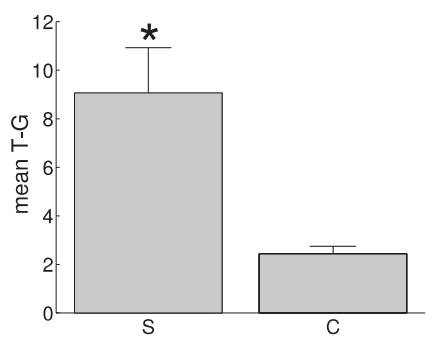

(a) Robustness to perturbation

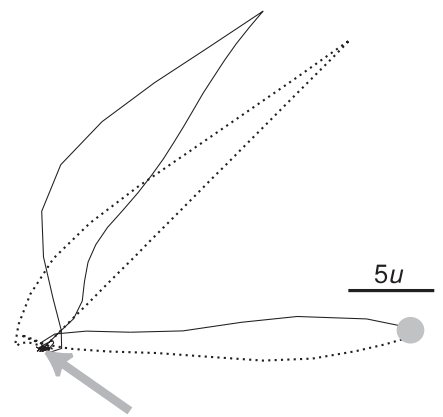

(b) S-network

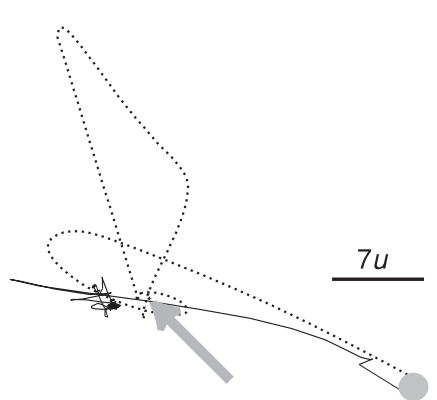

(c) C-network

Figure 3 Robustness of S-networks and C-networks to a novel perturbation: (a) Mean displacement of G from T during enforced $\mathrm{H}$ movement, calculated over the 20 time-steps of enforced movement and the 10 time-steps following for $10 \mathrm{~S}$-networks and $10 \mathrm{C}$-networks. Asterisk indicates statistical significance $(p<0.001$, two-tailed $t$ test). (b, c) Trajectories generated by a typical (b) S-network and (c) C-network during this operation (solid line $\mathrm{G}$, dotted line $\mathrm{H}$, gray circles indicates initial positions of $\mathrm{G}$ and $\mathrm{H}$, gray arrows indicate positions of $\mathrm{T}$ ). For the $\mathrm{S}$-network, fixation is lost (both $\mathrm{H}$ and $\mathrm{G}$ are displaced from the target), whereas for the $\mathrm{C}$-network, fixation is maintained ( $\mathrm{G}$ remains close to the target even though $\mathrm{H}$ is displaced). 

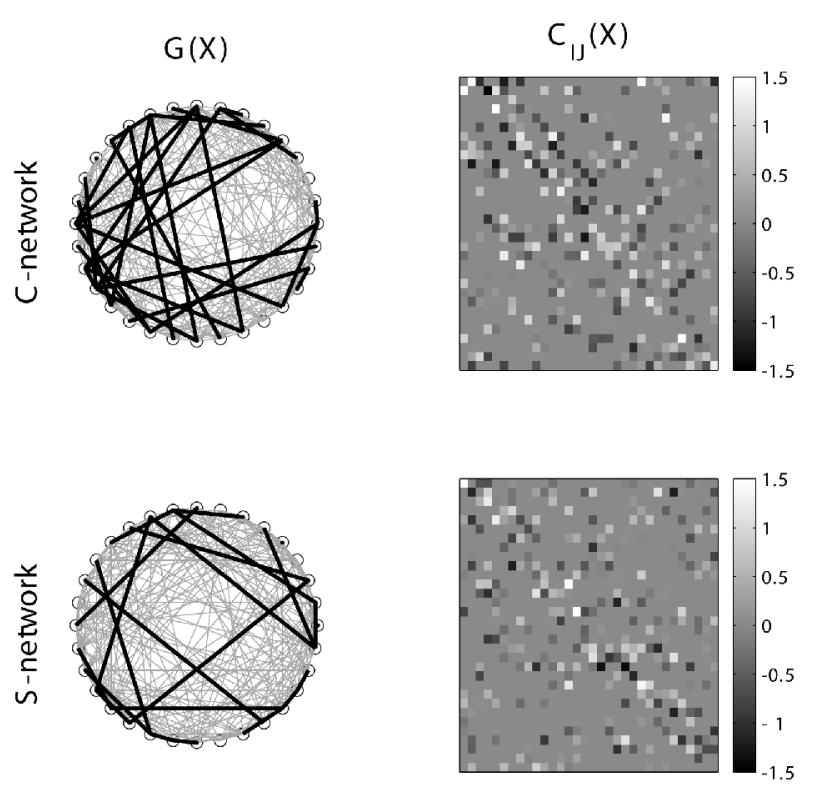

Figure 4 Example evolved networks. Top row: a C-network, bottom row: an S-network. $G(X)$ : networks represented as graphs, thin lines show feedforward connections, thick lines show reciprocal connections. $\mathbf{C}_{\mathrm{ij}}(\mathrm{X})$ : corresponding connectivity matrices. Vertices of each graph have been reordered so that vertices sharing many of the same connections are placed next to each other.

$\mathbf{A}_{\mathrm{ij}}(\mathrm{X})$ by replacing all non-zero elements in $\mathbf{C}_{\mathrm{ij}}(\mathrm{X})$ with the value 1 (we also tried various thresholds; see later). We calculated four graph-theoretic quantities from each $\mathbf{A}_{\mathbf{i j}}(\mathbf{X})$. The first, $f_{\text {recip }}$, measures the fraction of edges in $G$ for which a directly reciprocal connection exists. The "cluster index", $f_{\text {clust }}$, measures the fraction of possible connections among the immediate neighbors of a node that actually exist, averaged across all nodes (the immediate neighbors of a node $i$ are those nodes which are connected to $i$ by either an incoming or an outgoing edge). This quantity, introduced by Watts \& Strogatz (1998), is sometimes referred to as the "cliquishness" of a network. Low values of $f_{\text {recip }}$ and $f_{\text {clust }}$ indicate the absence of distinct groups of nodes. The diameter of $G$, diam ${ }_{G}$, is the maximum number of steps needed to reach any node $i$ from any other node $j$. It is computed by taking the maximum of the matrix $\mathbf{S}_{\mathrm{ij}}(\mathrm{X})$ which holds the lengths of the shortest paths linking elements $i$ and $j$. The global mean of $\mathbf{S}_{\mathrm{ij}}(\mathrm{X})$ is known as the characteristic path length $l_{\text {path }}$ of $G$ (Watts \& Strogatz, 1998). Low values for $\operatorname{diam}_{G}$ and $l_{\text {path }}$ indicate that no two nodes are separated by more than a few connections. Algorithms for computing all four of these metrics from adjacency matrices are described in Sporns (2002). Different network classes have distinct profiles when analyzed using these metrics. Random networks have low $f_{\text {recip }}$ and $f_{\text {clust }}$, as well as low $\operatorname{diam}_{G}$ and a short $l_{\text {path }}$. Socalled "small-world" networks are characterized by a

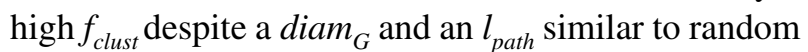
networks (Watts \& Strogatz, 1998).

Table 3 shows the results of applying these metrics to the fittest networks from each EA in each condition. Values were also calculated for an additional 64 networks with randomly assigned $\mathbf{C}_{\mathrm{ij}}(\mathrm{X})$ matrices (R-networks) with equivalent $N$ and $K$ (and a per-node in-degree of 8 ). The table indicates that there are no significant differences between any pair of conditions (including random) in any of the metrics. Why are no differences observed? It may be that the transformation of $\mathbf{C}_{\mathrm{ij}}(\mathrm{X})$ into $\mathbf{A}_{\mathrm{ij}}(\mathrm{X})$ overemphasizes the impor-

Table 3 Structural analysis of network types.

\begin{tabular}{lllll}
\hline Network type & $f_{\text {recip }}$ & diam $_{G}$ & $l_{\text {path }}$ & $f_{\text {clust }}$ \\
\hline S-network & $0.27(0.028)$ & $3.1(0.32)$ & $1.82(0.01)$ & $0.25(0.001)$ \\
T-network & $0.26(0.006)$ & $3.2(0.42)$ & $1.83(0.02)$ & $0.25(0.001)$ \\
H-network & $0.26(0.019)$ & $3.3(0.67)$ & $1.83(0.03)$ & $0.25(0.001)$ \\
C-network & $0.27(0.023)$ & $3.0(0.01)$ & $1.82(0.01)$ & $0.24(0.001)$ \\
R-network & $0.27(0.042)$ & $3.0(0.01)$ & $1.82(0.01)$ & $0.25(0.001)$ \\
\hline
\end{tabular}

$f_{\text {recip }}:$ the fraction of edges for which a directly reciprocal connection exists; $\operatorname{diam}_{G}:$ graph diameter; $l_{\text {path }}:$ characteristic path length; $f_{\text {clust }}$ : cluster index.

Each entry in the table shows the mean value with their standard error (in parentheses) calculated from 10 networks of each type (64 for R-networks). 
tance of weak connections. We tested this possibility by recalculating values after thresholding adjacency matrices to include only relatively strong connections. We tested a variety of thresholds, but in no case did we observe differences in structural measures among conditions (results not shown). This suggests that specific connection strengths may be critical for the behavioral properties of the networks. Importantly, this is not to say that network anatomy is irrelevant to the behavioral differences among network types; rather, it highlights the need for graph-theoretic methods that are directly applicable to weighted as well as to binary networks (see Section 4.1). ${ }^{2}$

\subsection{Dynamical analysis}

3.3.1 Complexity, Integration, and Entropy In order to characterize the dynamical properties of the evolved networks, we used a series of measures based on the statistical foundations of information theory. These measures were developed to assess quantitatively the complexity of the dynamics supported by neuroanatomical motifs characteristic of the cerebral cortex (Tononi et al., 1994; Sporns et al., 2000). An important advantage of these measures is that, unlike most structural analyses, they can be applied equally to both weighted and unweighted networks. A system can be considered to be complex when its individual elements interact, but do not act totally in concert, as in a crystal, nor, on the other hand, totally at random, as in an ideal gas. This idea has been refined into a definition of "neural complexity" $C(\mathrm{X})$ of a system $\mathrm{X}$ : a neurally complex system is one in which small subsets of the system show high statistical independence, but large subsets show low statistical independence (Tononi et al., 1994). In other words, such systems balance dynamical segregation-their component parts are differentiated - with dynamical integrationas larger and larger subsets of elements are considered, they become increasingly integrated. ${ }^{3}$ Other related dynamical measures are entropy $H(\mathrm{X})$, which characterizes the overall statistical independence of the elements of $\mathrm{X}$, and integration $I(\mathrm{X})$, which captures the overall degree to which the dynamics of a system deviate from statistical independence.

Calculation of these measures requires a knowledge of $\operatorname{COV}(\mathrm{X})$, the covariances of the elements of $X$. Under the assumptions that: (1) every node in the network has activity that can be described by a sta- tionary stochastic Gaussian process; (2) nodes implement linear input-output functions, $\operatorname{COV}(X)$ can be derived analytically from the corresponding connectivity matrix $\mathbf{C}_{\mathbf{i j}}(\mathrm{X})$ (Tononi et al., 1994):

$$
\operatorname{COV}(X)=\mathbf{Q}^{T} \times \mathbf{Q}, \quad \mathbf{Q}=\left[1-\mathbf{C}_{\mathrm{ij}}(\mathrm{X})\right]^{-1}
$$

where $T$ indicates matrix transpose. Full details for calculating $C(\mathrm{X}), I(\mathrm{X})$, and $H(\mathrm{X})$, given $\operatorname{COV}(\mathrm{X})$, are provided in Appendix B.

An important property of the analytical step shown by Equation 3 is that $\operatorname{COV}(\mathrm{X})$, and therefore also the resulting values of $C(\mathrm{X}), I(\mathrm{X})$, and $H(\mathrm{X})$, are independent of behavioral context. This method is therefore best described as characterizing the intrinsic dynamical properties of a network $\mathrm{X}$.

3.3.2 Intrinsic Dynamics For each EA in each condition, the fittest member of the final generation was used to generate a covariance matrix (Equation 3), which in turn was used to calculate the intrinsic neural complexity $C(\mathrm{X})$, integration $I(\mathrm{X})$, and entropy $H(\mathrm{X})$ of the network dynamics. Values were also calculated for 64 R-networks.

Figure 5 shows mean values and standard deviations of intrinsic $C(\mathrm{X}), I(\mathrm{X})$, and $-H(\mathrm{X})$, for each network type. In contrast to the structural analysis, clear differences are evident: random R-networks scored lowest on all three dynamical measures, and C-networks scored highest (note that we plot $-H(\mathrm{X})$ rather than $H(\mathrm{X})$ ). Differences between $\mathrm{C}$-networks and all other network types were significant at the $p<0.01$ level by two-tailed $t$ test for $C(\mathrm{X}), I(\mathrm{X})$, and $H(\mathrm{X})$. These observations indicate that adaptation to comparatively complex environmental/phenotypic conditions $\left(E_{C}\right)$ endows C-networks with above-random neural complexity and integration, and below-random entropy. Furthermore, only networks with these dynamical characteristics displayed the behavioral robustness and flexibility described in Section 3.1.

Figure 5 also shows that $\mathrm{S}$-networks, H-networks, and $\mathrm{T}$-networks scored at intermediate levels on all measures. Values of $C(\mathrm{X}), I(\mathrm{X})$ and $-H(\mathrm{X})$ for these networks are significantly lower than the corresponding values for $\mathrm{C}$-networks, and significantly higher than the corresponding values for R-networks $(p<$ 0.01 , two-tailed $t$ test); there are no significant differences in $C(\mathrm{X}), I(\mathrm{X})$ or $-H(\mathrm{X})$ among S-networks, 

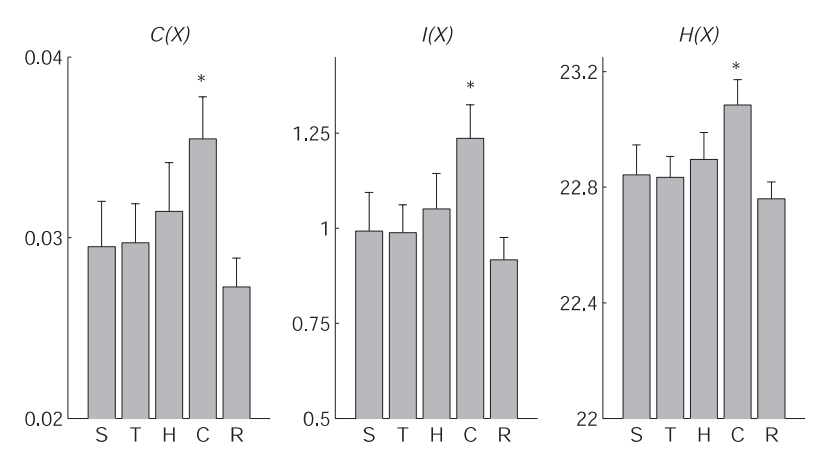

Figure 5 Mean and standard error intrinsic $C(\mathrm{X}), I(\mathrm{X})$, and $-H(\mathrm{X})$ for S-networks (S), T-networks (T), H-networks (H), Cnetworks $(\mathrm{C})$, and R-networks $(\mathrm{R})$. Asterisks indicate that the value for $\mathrm{C}$-networks is significantly higher than for all other network types ( $p<0.01$, two-tailed $t$ tests). Values of $C(\mathrm{X}), I(\mathrm{X})$ and $-H(\mathrm{X})$ for S-networks, T-networks, and H-networks are significantly lower than the corresponding values for C-networks, and significantly higher than the corresponding values for $\mathrm{R}$-networks ( $p<0.01$, two-tailed $t$ tests). There are no significant differences in $C(\mathrm{X}), I(\mathrm{X})$ or $-H(\mathrm{X})$ among S-networks, T-networks, and $\mathrm{H}$-networks. All distributions are normal ( $p<0.05$, Bera-Jarques test).

T-networks, and H-networks. Changes in intrinsic dynamics, therefore, depend both on properties of the environment (condition $E_{T}$ ) and on properties of the phenotype (condition $E_{H}$ ).

While these results show that neurally complex network dynamics can accompany adaptive behavior in rich environmental/phenotypic conditions, intrinsic neural complexity is not unique in this respect, since similar patterns of results are apparent for both intrinsic integration $I(\mathrm{X})$ and entropy $H(\mathrm{X})$.

3.3.3 Interactive Dynamics The interactive dynamics of a network in a given behavioral context are calculated using observations of network activity during behavior. In the present study, each behavioral trial yields a matrix $\mathrm{F}$ that contains the activity of each node over all 600 time-steps. This matrix can be used to generate a covariance matrix according to:

$$
\operatorname{COV}(\mathrm{X})=\operatorname{cov}(\dot{\mathbf{F}})
$$

where $\dot{\mathbf{F}}$ is the first derivative (with respect to time) of the activity matrix and $\operatorname{cov}()$ is a standard covariance function. This approach was chosen because rates of change of node activities are (i) more likely to satisfy assumptions of stationarity (Hamilton, 1994), and (ii)

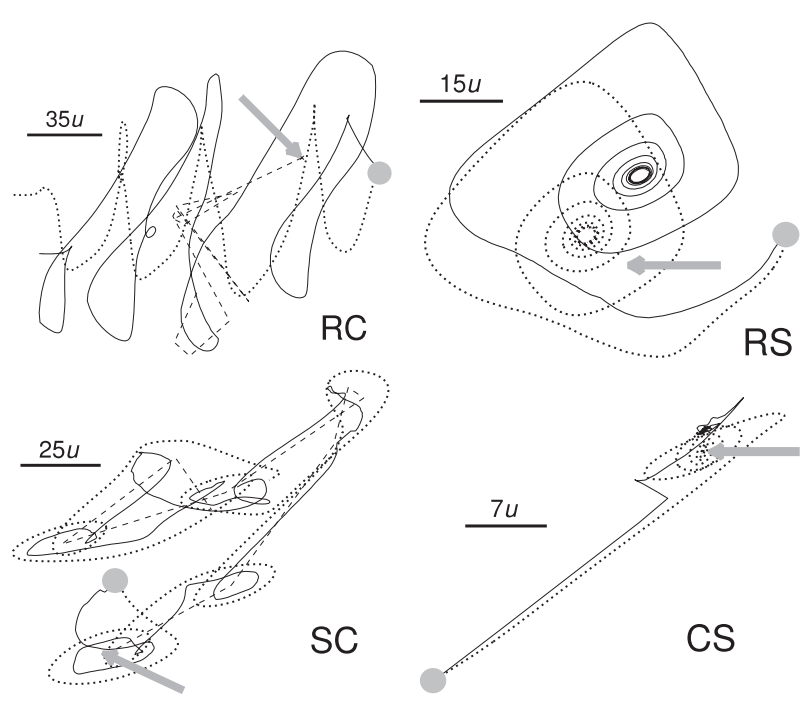

Figure 6 Representative behavior for various networkenvironment/phenotype combinations, showing trajectories of $\mathrm{G}$ (solid line), $\mathrm{H}$ (dotted line), and $\mathrm{T}$ (dashed line), gray circles indicate initial positions of $G$ and $H$, gray arrows indicate initial positions of T. RC: R-network in condition $E_{C}$; RS: R-network in condition $E_{S}$; SC: S-network in condition $E_{C}$; CS: C-network in condition $E_{S}$. Trajectories of a $\mathrm{C}$-network in condition $E_{C}$ and an S-network in condition $E_{S}$ are shown in Figure 2.

better reflect interactions between the network and its phenotype and environment than do absolute activity levels. $\operatorname{COV}(\mathrm{X})$ can then be used to calculate corresponding values of interactive $C(\mathrm{X}), I(\mathrm{X})$, and $H(\mathrm{X})$ in just the same way as for the calculation of intrinsic dynamics (see Appendix B). Note that calculation of interactive dynamics does not require Equation 3, and so does not assume linear system dynamics.

We compared interactive dynamics for C-networks, S-networks, and random R-networks evaluated in both $E_{C}$ and $E_{S}$ conditions, recording both network activities and behavioral trajectories. Figure 6 shows representative trajectories from the various combinations (trajectories of a C-network in condition $E_{C}$ and an S-network in condition $E_{S}$ are shown in Figure 2). R-networks in $E_{C}$ and $E_{S}$ conditions never achieve fixation, and their behavior is highly variable. S-networks in $E_{C}$ conditions are unable to achieve or maintain fixation despite a general tendency to track towards the target, and C-networks in $E_{S}$ conditions rapidly achieve fixation despite some persistent oscillation in the head direction. Notice that in all except the last of these cases, the behavioral dynamics are rich, even though the behavior itself is not adaptive. 

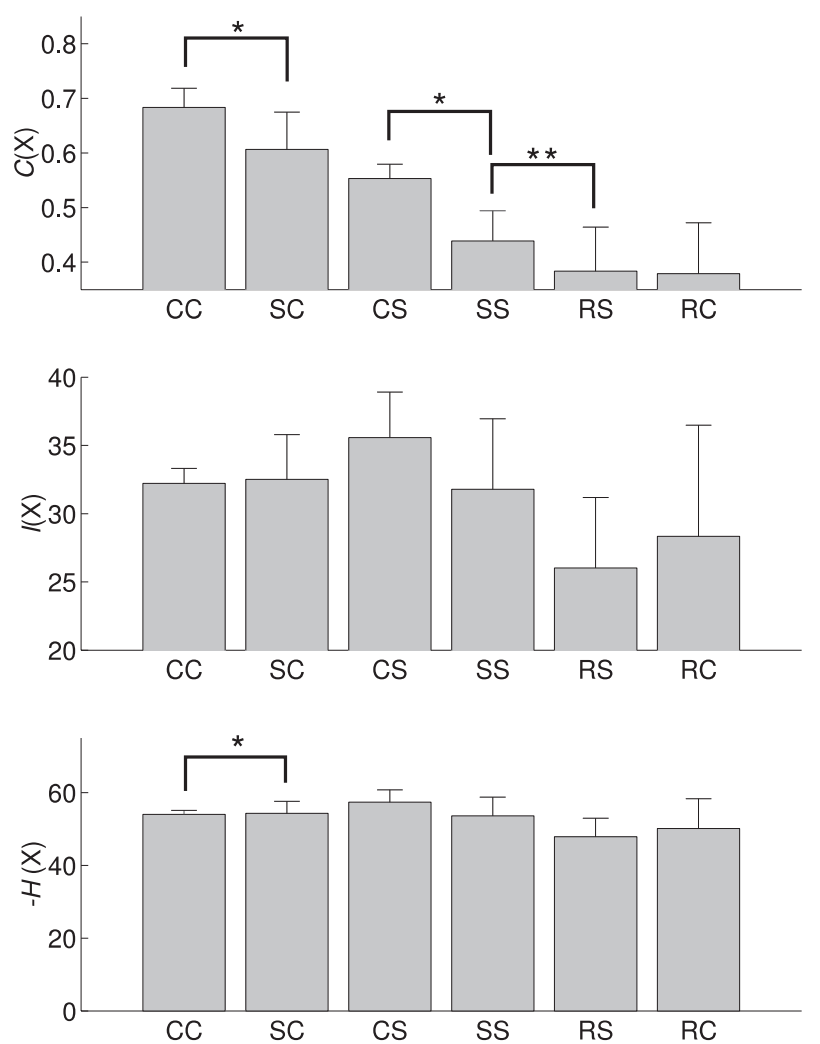

Figure 7 Interactive $C(\mathrm{X}), I(\mathrm{X})$, and $-H(\mathrm{X})$ calculated from covariance matrices derived from recorded neural activity during behavior. CC: C-network in condition $E_{C}$; SC: S-network in condition $E_{C}$; CS: C-network in condition $E_{S}$; SS: S-network in condition $E_{S}$; RS: R-network in condition $E_{S}$; RC: R-network in condition $E_{C}$. Each column shows mean and standard error calculated from 10 repetitions of each combination of network and condition. There is a significant correlation between the rank ordering of combination (from "CC" to "RC") and mean $C(\mathrm{X})(r$ $=0.97, p<0.01$; Spearman's rank correlation). No significant correlations exist for $I(\mathrm{X})$ or $-H(\mathrm{X})(p>0.1)$. Asterisks indicate statistically significant differences between combinations $\left({ }^{\star} p<0.01,{ }^{* \star} p<0.05\right.$, two-tailed $t$ test; only adjacent combinations were tested). Note that values of interactive $C(\mathrm{X})$ are greater than intrinsic $C(\mathrm{X})$. This may be due to the greater range of covariance values in the former case (results not shown). All distributions are normal ( $p<0.05$, Bera-Jarques test).

Figure 7 shows the interactive dynamics generated in each combination of network and condition. The top row shows a clear gradient in interactive $C(\mathrm{X})$ proceeding from C-networks in condition $E_{C}$ ("CC"; high), to R-networks in conditions $E_{S}$ and $E_{C}$ ("RS", "RC"; low). Intermediate values of $C(\mathrm{X})$ were observed for simple networks in rich environmental/ phenotypic conditions ("SC"), and for complex net- works in simple conditions ("CS"). C-networks in condition $E_{C}$ ("CC") have significantly higher $C(\mathrm{X})$ than all other combinations. The remaining significant differences among combinations are also consistent with the steady gradient in interactive $C(\mathrm{X})$.

Strikingly, only $C(\mathrm{X})$ shows a pattern of values reflecting rich adaptive behavior. Neither $I(\mathrm{X})$ (Figure 7, middle row) nor $H(\mathrm{X})$ (bottom row) show any such sensitivity. This is in contrast to the intrinsic analysis, in which all three dynamical measures behaved in a similar way (Figure 5).

It is also notable that R-networks evoke low interactive $C(\mathrm{X})$ despite displaying the rich behavioral patterns shown in Figure 6. The contrast between Rnetworks and C-networks, together with the steady gradient in interactive $C(\mathrm{X})$ across conditions (Figure 7 , top row), suggests that $C(\mathrm{X})$ is indeed selectively sensitive to the dynamics of rich adaptive behavior. Figure 7 also suggests that high interactive $C(\mathrm{X})$ depends on a combination of environmental and phenotypic properties. The intermediate values of $C(\mathrm{X})$ in Figure 7 ("SC" and "CS") are consistent with the intermediate values of intrinsic $C(\mathrm{X})$ associated with rich environments (condition $E_{T}$ ) and complex phenotypes (condition $E_{H}$ ) shown in Figure 5.

Overall, the unique correspondence of interactive $C(\mathrm{X})$ with adaptive behavior in rich environmental/ phenotypic conditions suggests that it may indeed reflect a common property of the functional organization of adaptive neural systems.

\section{Discussion}

This paper has described a novel methodology able to relate, quantitatively, behavioral complexity and environmental complexity to the "neural" complexity of the underlying mechanisms, where neural complexity reflects an even balance between integration and segregation in the dynamics of a network. Applying this methodology to the evolution of neural network controllers in a target fixation task, we found that adaptive behavior in a rich environmental/phenotypic conditions was accompanied by neurally complex network dynamics.

Specifically, we used evolutionary algorithms to generate neural networks able to support target fixation in environmental/phenotypic conditions of qualitatively different levels of complexity. We found 
that those networks that evolved in rich conditions exhibited (qualitatively) more complex behavior than networks that evolved in comparatively simple conditions (Figure 2). When compared in an environment involving a novel perturbation, networks that evolved in rich conditions showed greater robustness than networks that evolved in simple conditions (Figure 3).

This robustness was reflected by significantly higher neural complexity $C(\mathrm{X})$ for networks which evolved in rich environmental/phenotypic conditions, than for networks which evolved in relatively simple conditions, or for networks with the same number of nodes and edges but with randomly arranged connectivity (Figures 5 and 7). This was true for both intrinsic and interactive methods of calculating dynamics, where the former are derived analytically from network connectivity, and the latter are computed from observed network activity during behavior. While intrinsic dynamics did not differentiate between neural complexity $C(\mathrm{X})$, integration $I(\mathrm{X})$, or entropy $H(\mathrm{X})$ (Figure 5), interactive dynamics revealed that only $C(\mathrm{X})$ was consistently associated with adaptive behavior in rich conditions (Figure 7). Interactive $I(\mathrm{X})$ and $H(\mathrm{X})$ did not show any reliable correlation with adaptive behavior or environmental complexity.

Both types of dynamical analysis indicate that the magnitude of neural complexity depended on a combination of environmental, phenotypic, and mechanistic properties. Networks evolved in conditions of intermediate richness (conditions $E_{T}$ and $E_{H}$ ) generated intermediate values of intrinsic $C(\mathrm{X})$ (Figure 5). Networks evolved in rich conditions and tested in simple conditions, and vice versa, generated intermediate values of interactive $C(\mathrm{X})$ (Figure 7).

Perhaps surprisingly, structural analysis was not sufficient to distinguish among networks with different behavioral profiles. One possible reason for this is that structural analysis is usually best suited to binary matrices, and it may be that binary matrices are in general insufficiently sensitive to functional differences in the corresponding weighted networks. It is a major advantage of dynamical analysis, both intrinsic and interactive, that it can be applied equally to both weighted and unweighted networks.

Taken together these results show that, in the present target fixation task, neural complexity is selectively sensitive to the dynamics of adaptive behavior in a rich environmental/phenotypic conditions. While this correspondence may not hold for all instances of complex behavior (Braitenberg, 1984; Simon, 1988), these findings are consistent with the proposal that $C(\mathrm{X})$ may reflect a common property of the functional organization of adaptive neural systems.

Previous work using $C(\mathrm{X}), I(\mathrm{X})$, and $H(\mathrm{X})$ has focused on the relationship between structure and dynamics in the absence of behavior. For example, Sporns et al. (2000) used evolutionary algorithms to automatically specify networks good at generating each class of intrinsic dynamics. Very different graph structures evolved in each case, and, when compared with graphs based on known cortical connection matrices (of macaque monkey and cat cortex), those that generated high values of $C(\mathrm{X})$ provided the closest match. Moreover, when the intrinsic dynamical properties of the known cortical connection matrices were analyzed, near-optimal values of $C(\mathrm{X})$ were found. These findings are consistent with the idea that high complexity is associated with the adaptive transformation of input to output. The present results complement these observations by explicitly instantiating networks in environments in which these transformations actually happen. The present study also introduces the distinction between intrinsic and interactive dynamics. Interactive dynamics offers several advantages over intrinsic dynamics:

- Calculation of intrinsic dynamics requires the assumptions that: (1) every node in the network is activated by Gaussian noise; (2) nodes implement linear input-output functions (see Section 3.1.1). Calculation of interactive dynamics does not require these assumptions (although one must still assume stationarity; see Appendix B).

- Interactive dynamics can be calculated for networks that undergo structural changes over time. For example, networks that make use of shortterm synaptic plasticity have the property that the corresponding $\mathbf{C}_{\mathrm{ij}}(\mathrm{X})$ matrices are changing on the same timescale as behavior (Floreano \& Mondada, 1996). Calculation of the intrinsic dynamics would face the problem of choosing which $\mathbf{C}_{\mathrm{ij}}(\mathrm{X})$ matrix to use a basis for deriving a covariance matrix, but interactive dynamics does not face this problem: covariance matrices can be derived explicitly from the activity of the network over time. 
- The same approach could be extended to networks that are not fully determined by their nodes and edges, for example the so-called "GasNets" (Husbands, Smith, Jakobi, \& O'Shea, 1998; Read Montague, Gally, \& Edelman, 1991), which involve spatial diffusion of simulated neuromodulatory substances. It may even be possible to calculate interactive dynamics when the structure of the underlying network is not fully known.

- Interactive dynamics enables the comparison of dynamics generated by a particular network in different behavioral or environmental situations. The present study has used such comparisons to show that interactive dynamics can be more sensitive than intrinsic dynamics to neural processes underlying rich adaptive behavior.

In certain cases intrinsic dynamics may remain preferable. Calculation of intrinsic dynamics is usually computationally cheaper than calculation of interactive dynamics. In addition, intrinsic dynamics are in a sense more general than interactive dynamics, precisely because they characterize the dynamical potential of a network in the absence of behavioral context. It should nonetheless be emphasized that both intrinsic and interactive dynamical methods offer significant advantages over structural methods. Not only do dynamic methods apply equally to both weighted and unweighted networks, but dynamic analyses go directly to the heart of the problem of network analysis, which is to understand how networks behave (Newman, 2003).

Despite the increasing popularity of evolutionary design of neural systems, standard techniques for their analysis remain sparse (Nolfi \& Floreano, 2000). One promising avenue, complementary to the present approach, is "dynamical systems analysis" (Abraham \& Shaw, 1981; Strogatz, 1994; Port \& van Gelder, 1995). Dynamical systems analysis typically uses differential (or difference) equations to describe the evolution of a system in terms of trajectories in a phase space, the dimensions of which are determined by the variables of interest in the system. While applications of these techniques to evolved neural systems have illuminated dynamical features that underlie simple examples of adaptive behavior (Beer \& Gallagher, 1992; Beer, Chiel, \& Gallagher, 1999; Chiel, Beer, \& Gallagher, 1999; Smith, Husbands, Philippides, \& O'Shea, 2002; Beer, 2004), such insights do not come easily, and the required depth of analysis may complicate the extension of such methods to larger neural systems.

The present methods describe a narrower approach: the extraction of certain statistically defined properties from representations of the stationary dynamical relations among system elements (covariances). While this approach does not reveal specific dynamical features, it allows the easy comparison of distinct systems, its application to empirical data is straightforward (see later), and it focuses on global dynamical properties that have a previous theoretical grounding in terms of dynamical integration, segregation, and the balance between the two.

\subsection{Future directions}

A natural extension is to ask why interactive $C(\mathrm{X})$ associates more strongly with environmental and behavioral complexity than does intrinsic $C(\mathrm{X})$. It may make intuitive sense for this to be so, since the environment, behavior, and mechanism are always tightly coupled in natural situations; however, a full answer will require analysis of the specific causal transactions that underlie the behavioral differences among network types. This would likely involve following the dynamics in each node as well as the network dynamics generated by their mutual interactions. Future research should therefore explore-perhaps using dynamical systems analysis-how the behavioral phenomena in the various network/condition combinations relate to the balance between dynamical segregation and integration that is measured by $C(\mathrm{X})$. It could also be asked whether behavioral robustness can be instilled in a network by selecting for task performance and high $C(\mathrm{X})$ concurrently during evolution. Finally, to test the generality of the present conclusions, it would be valuable to explore the consequences of high $C(\mathrm{X})$ across a broad range of network types and task conditions.

More generally, the present methods are not restricted to artificial networks. At the forefront of experimental neuroscience is the characterization of dynamics of biological neural networks in awake, behaving animals. Multielectrode technology has now reached the point at which the activities of hundreds of neurons can be recorded simultaneously, from different areas of the brain, while an animal is behaving (Csicsvari et al., 2003). Measuring the complexity, 
integration, and entropy of such data will allow, among other things, direct testing of the hypothesis that complex behaviors incur underlying neural dynamics of high neural complexity. Importantly, such dynamics would necessarily be interactive; in general the corresponding intrinsic dynamics could not be calculated since the structure of the underlying network would not be known.

Along different lines, the development of analytical tools able to characterize structural properties of graphs with weighted edges remains a significant challenge (although see Latora \& Marchiori, 2001; Newman, 2001; Cannings \& Penman, 2002). It is possible that the development and application of such tools would allow the various kinds of networks described in this study to be distinguished by structural criteria alone. Another general challenge is to understand in greater depth the relations between intrinsic dynamics, interactive dynamics, and network structure. For example, it may be important to know what, if anything, can be inferred about the structure of a network given the interactive dynamics of a subset of its elements. Finally, we still lack adequate quantitative measures for environmental and behavioral complexity. The development of such measures would allow the testing of hypotheses of greater specificity by the present methodology.

The quantitative characterization of network dynamics is becoming increasingly important across a broad range of domains (Albert \& Barábasi, 2002; Newman, 2003). The present results are consistent with the idea that high "neural" complexity may reflect a common property of the functional organization of complex adaptive systems such as networks. These findings may therefore contribute to the analysis and design of network systems of high robustness and flexibility.

\section{Notes}

1 We used a distributed evolutionary algorithm (Mitchell, 1997): each EA was initialized by arranging the population on an $8 \times 8$ toroidal grid and evaluating the fitness of each network. Each subsequent generation involved 64 repeats of the following: a random grid position was chosen determining a $3 \times 3$ sub-grid. Stochastic rank-based selection was then used to select a weak member of this sub-grid for replacement, and a strong member as the "parent." A mutated copy of the parent then replaced the weak member and was evaluated (the parent was also reevaluated with probability 0.75 ).

2 In support of this interpretation, we were not able to evolve unweighted networks for the present target fixation tasks.

3 While neural complexity is very different from algorithmic (Kolmogorov) complexity, which is maximal in the random limit, it bears some similarity to recent complexity metrics that are maximal for systems intermediate between randomness and regularity, for example "statistical complexity" (Crutchfield \& Young, 1995).

\section{Acknowledgments}

This research was made possible by the Neurosciences Research Foundation, which supports the work of The Neurosciences Institute. We are grateful to Drs J. Gally, J. Iversen, E. Izhikevich, J. Krichmar, and J. McKinstry, and to our reviewers for detailed criticism. Special thanks to Bruno van Swinderen for Figure 1.

\section{References}

Abraham, R., \& Shaw, C. (1981). Dynamics, the geometry of behavior, parts 1-4. Santa Cruz, CA: Aerial Press.

Albert, R., \& Barábasi, A. (2002). Statistical mechanics of complex networks. Reviews of Modern Physics, 74, 47-97.

Beer, R. (2004). The dynamics of active categorical perception in an evolved model agent. Adaptive Behavior (in press).

Beer, R., Chiel, H., \& Gallagher, J. (1999). Evolution and analysis of model CPGs for walking II: General principles and individual variability. Journal of Computational Neuroscience, 7(2), 119-147.

Beer, R., \& Gallagher, J. (1992). Evolving dynamical neural networks for adaptive behavior. Adaptive Behavior, 1, 94110 .

Bollobás, B. (1985). Random graphs. Academic Press, London.

Braitenberg, V. (1984). Vehicles: experiments in synthetic psychology. MIT Press, Cambridge, MA.

Cannings, C., \& Penman, C. (2002). Random graphs. In C. Rao \& D. Shanbhag, D. (Eds.), Stochastic processes: modelling and simulation, Vol. 21. Handbook of statistics series. Amsterdam: Elsevier.

Chiel, H., Beer, R., \& Gallagher, J. (1999). Evolution and analysis of model CPGs for walking I: Dynamical modules. Journal of Computational Neuroscience, 7(2), 99-118. 
Crutchfield, J., \& Young, K. (1995). Inferring statistical complexity. Physical Review E, 59, 275-283.

Csicsvari, J., Henze, D., Jamieson, B., Harris, K., Sirota, A., Bartho, P., Wise, K., \& Buzsaki, G. (2003). Massively parallel recording of unit and local field potentials with silicon-based electrodes. Journal of Neurophysiology, 90(2), 1314-1323.

Di Paolo, E., Noble, J., \& Bullock, S. (2000). Simulation models as opaque thought experiments. In M. Bedau, J. McCaskill, N. Packard, \& S. Rasmussen (Eds.), Artificial Life VII: Proceedings of the Seventh International Conference on the Simulation and Synthesis of Living Systems (pp. 497-506). Cambridge, MA: MIT Press.

Fletcher, J., Zwick, M., \& Bedau, M. (1998). Effect of environmental texture on evolutionary adaptation. In C. Adami, R. Belew, H. Kitano, \& C. Taylor (Eds.), Proceedings of the Sixth International Conference on Artificial Life (pp. 189-199). Cambridge, MA: MIT Press.

Floreano, D., \& Mondada, F. (1996). Evolution of plastic neurocontrollers for situated agents. In P. Maes, M. Mataric, J. Meyer, J. Pollack, \& S. Wilson, (Eds.), From Animals to Animats 4: Proceedings of the Fourth International Conference on the Simulation of Adaptive Behavior (pp. 401410). Cambridge, MA: MIT Press.

Godfrey-Smith, P. (1996). Complexity and the function of mind in nature. Cambridge: Cambridge University Press.

Hamilton, J. (1994). Time series analysis. Princeton, NJ: Princeton University Press.

Husbands, P., Smith, T., Jakobi, N., \& O'Shea, M. (1998). Better living through chemistry: Evolving GasNets for robot control. Connection Science, 10(3/4), 185-211.

Kaufman, S. (1993). The origins of order. Self-organisation and selection in evolution. Oxford: Oxford University Press.

Latora, V., \& Marchiori, M. (2001). Effcient behavior of smallworld networks. Physical Review Letters, 87(19), 198701.

Lindgren, K. (1991). Evolutionary phenomena in simple dynamics. In C. Langton, J. Farmer, S. Rasmussen, \& C. Taylor (Eds.), Artificial life II, vol. XVI of Santa Fe Institute studies in the sciences of complexity (pp. 295-312). Reading, MA: Addison-Wesley.

Menczer, F., \& Belew, R. (1996). From complex environments to complex behaviours. Adaptive Behavior, 4(3/4), 309-355.

Mitchell, M. (1997). An introduction to genetic algorithms. Cambridge, MA: MIT Press.

Newman, M. (2001). Who is the best connected scientist? A study of scientific coauthorship networks. Physical Review $E, 64,016131$. cond-mat/0011144.

Newman, M. (2003). The structure and function of complex networks. SIAM Review, 45(2), 167-256.

Nolfi, S., \& Floreano, D. (2000). Evolutionary robotics: the biology, intelligence, and technology of self-organizing machines. Cambridge, MA: MIT Press.
Papoulis, A., \& Pillai, S. (2002). Probability, random variables, and stochastic processes (4th ed.). New York: McGraw-Hill.

Port, R., \& van Gelder, T. (1995). Mind as motion: explorations in the dynamics of cognition. Cambridge, MA: MIT Press.

Read Montague, P., Gally, J., \& Edelman, G. (1991). Spatial signalling in the development and function of neuronal connections. Cerebral Cortex, 1, 199-220.

Seth, A. (1997). Interaction, uncertainty, and the evolution of complexity. In P. Husbands \& I. Harvey (Eds.), Proceedings of the Fourth European Conference on Artificial Life (pp. 521-530). Cambridge, MA: MIT Press.

Seth, A. (1998). The evolution of complexity and the value of variability. In C. Adami, R. Belew, H. Kitano, \& C. Taylor (Eds.), Artificial Life VI: Proceedings of the Sixth International Conference on the Simulation and Synthesis of Living Systems (pp. 209-221). Cambridge, MA: MIT Press.

Seth, A. (2000). On the relations between behaviour, mechanism, and environment: explorations in artificial evolution. $\mathrm{PhD}$ Thesis, University of Sussex.

Shannon, C., \& Weaver, W. (1949). The mathematical theory of communication. Urbana, IL: University of Illinois Press.

Shibata, T., Vijayakumar, S., Conradt, J., \& Schaal, S. (2001). Biomimetic oculomotor control. Adaptive Behavior, 9(4), 189-209.

Simon, H. (1988). The sciences of the artificial (3rd ed.). Cambridge, MA: MIT Press.

Smith, T., Husbands, P., Philippides, A., \& O'Shea, M. (2002). Neuronal plasticity and temporal adaptivity: GasNet robot control networks. Adaptive Behavior, 10(3/4), 161183.

Sporns, O. (2002). Graph theory methods for the analysis of neural connectivity patterns. In R. Kötter (Ed.), Neuroscience databases. A practical guide (pp. 169-183). Boston, MA: Kluwer Publishers.

Sporns, O., Tononi, G., \& Edelman, G. (2000). Theoretical neuroanatomy: Relating anatomical and functional connectivity in graphs and cortical connection matrices. Cerebral Cortex, 10, 127-141.

Strogatz, S. (1994). Nonlinear dynamics and chaos. Cambridge, MA: Perseus Books.

Thompson, W. (1917). On growth and form. Cambridge: Cambridge University Press.

Todd, P., \& Yanco, H. (1996). Environmental effects on minimal behaviors in the minimat world. Adaptive Behavior, 4(3/4), 365-413.

Tononi, G., Sporns, O., \& Edelman, G. (1994). A measure for brain complexity: Relating functional segregation and integration in the nervous system. Proceedings of the National Academy of Science USA, 91, 5033-5037. 
Watts, D., \& Strogatz, S. (1998). Collective dynamics of "small world" networks. Nature, 393, 440-442.

Young, R. (1970). Mind, brain, and adaptation in the nineteenth century. Oxford: Clarendon Press.

\section{Appendix A: Model Implementation}

This appendix describes how the head direction projection $(\mathrm{H})$, the eye direction $(\mathrm{E})$, the gaze direction projection $(\mathrm{G})$ and the target $(\mathrm{T})$ are updated at each time-step. Two of the four output nodes influence $\mathrm{H}$ velocity in the $x, y$ plane $\left(s_{h x}, s_{h y}\right)$, and two infuence the velocity of $\mathrm{E}\left(s_{e x}, s_{e y}\right)$. These are used to update the velocities of $\mathrm{H}$ and $\mathrm{E}$ according to:

$$
\begin{aligned}
& V_{h x}(t)=m_{H} V_{h x}(t-1)+A_{H}\left(1-m_{H}\right) s_{h x}(t-\text { lag }) \\
& V_{h y}(t)=m_{H} V_{h y}(t-1)+A_{H}\left(1-m_{H}\right) s_{h y}(t-\text { lag }) \\
& V_{e x}(t)=m_{E} V_{e x}(t-1)+A_{E}\left(1-m_{E}\right) s_{e x}(t) \\
& \mathrm{V}_{e y}(\mathrm{t})=m_{E} V_{e y}(t-1)+A_{E}\left(1-m_{E}\right) s_{e y}(t)
\end{aligned}
$$

where $V_{h x}(t), V_{h y}(t)$ represent the velocity of $\mathrm{H}$ in the $x$, $y$ directions respectively at time $t$, and $V_{e x}(t), V_{e y}(t)$ represent the velocity of $\mathrm{E}$. In all cases, $V(t)$ is bounded by the corresponding value of $V_{\max }$. These values, as well as those of $A_{H}, A_{E}, m_{H}, m_{E}$, and lag are specified for each condition in Table 1 .

The positions of $\mathrm{H}, \mathrm{E}$, and $\mathrm{G}$ in the $x, y$ plane are updated at each time-step using:

$$
\begin{aligned}
& \mathrm{H}(t)=\mathrm{H}(t-1)+V_{h x}(t)+V_{h y}(t) \\
& \mathrm{E}(t)=\mathrm{E}(t-1)+V_{e x}(t)+V_{e y}(t) \\
& \mathrm{G}(t)=\mathrm{H}(t)+\mathrm{E}(t)
\end{aligned}
$$

where $\mathrm{H}(t)$ and $\mathrm{G}(t)$ represent the positions of $\mathrm{H}$ and $\mathrm{G}$ on the $x, y$ plane at time $t$, and $\mathrm{E}(t)$ represents the position of the eye relative to $\mathrm{H}$. If the distance between $\mathrm{G}$ and $\mathrm{H}$ exceeds $35.0 u$ then $\mathrm{G}$ is not updated at that time-step.

In conditions $E_{T}$ and $E_{C}$ the target position (T) is also updated at each time-step, alternating between 50 time-steps of drift and 50 time-steps without drift. Each period of drift is in a random direction at a random speed in the range 0.5 to 1.5 ( $u$ per time-step). During the intervening periods $\mathrm{T}$ is stationary except for occa- sional jumps (with a probability of 0.025 per time-step), each to a randomly chosen location within a radius of $25 u$ to $31 u$ of its previous location. T cannot leave the $100^{2} u^{2}$ area: If drift is leading it out of bounds the appropriate velocity component is reversed at the boundary.

\section{Appendix B: Calculation of Dynamics}

This appendix describes how to calculate entropy $H(\mathrm{X})$, integration $I(\mathrm{X})$, and neural complexity $C(\mathrm{X})$, given the covariance matrix $\operatorname{COV}(X)$ of a network $X$.

The magnitude of neural complexity can be estimated by asking how much the activity of different subsets of elements depends on other subsets. This is a relationship captured by the information-theoretic quantity of mutual information (MI), which is the amount of information that is redundant when two subsets $\mathrm{A}$ and $\mathrm{B}$ of a system are combined to form $\mathrm{AB}$ :

$$
M I(\mathrm{~A} ; \mathrm{B})=H(\mathrm{~A})+H(\mathrm{~B})-H(\mathrm{AB})
$$

where $H(\mathrm{~A})$ denotes the entropy of subset $\mathrm{A}$, which is a measure of its overall degree of statistical independence, the number of "yes/no" questions needed to fully know its state. Assuming stationarity, $H(\mathrm{X})$ of a system $X$ can be calculated from the standard formula (Papoulis \& Pillai, 2002):

$$
H(\mathrm{X})=0.5 \ln \left((2 \pi e)^{N}|\mathbf{C O V}(\mathrm{X})|\right)
$$

where $\operatorname{COV}(\mathrm{X})$ is the $N \times N$ covariance matrix of $\mathrm{X}$, and I.I denotes the matrix determinant. When calculating interactive dynamics, stationarity can be approached by taking the first derivative of the node activity matrix (Hamilton, 1994).

The neural complexity $C(\mathrm{X})$ of a system $\mathrm{X}$ can be calculated by summing the average MI between subsets of different sizes, for all possible bipartitions of the system:

$$
C(\mathrm{X})=\sum_{k=1}^{n_{t} / 2}\left\langle M I\left(\mathrm{X}_{j}^{k} ; X-\mathrm{X}_{j}^{k}\right)\right\rangle
$$

where $n_{t}$ is the total number of ways of bipartitioning $\mathrm{X}, \mathrm{X}_{j}^{K}$ is the $j$ th bipartition of size $k$, and $\langle$.$\rangle is the$ average across index $j$. Full calculation of $C(\mathrm{X})$ is computationally challenging because of the large 
number of bipartitions that are possible for any network of reasonable size. Here, we follow previous usage of these measures (Tononi et al., 1994; Sporns et al., 2000) by examining only partitions that divided $\mathrm{X}$ into pairs comprising a single node and the set of all remaining nodes.

Given $H(\mathrm{X})$, it is also possible to calculate the integration $I(\mathrm{X})$ of a system, which captures the overall degree to which the dynamics of a system deviate from statistical independence:

$$
I(\mathrm{X})=\sum_{i=1}^{N} H\left(x_{i}\right)-H(\mathrm{X})
$$

It is important to assure that observed changes in these measures were due to changes in covariance between elements, and not due to changes in variance. To achieve this, when calculating intrinsic dynamics we followed Sporns et al. (2000) by first dividing each element of $\mathbf{C}_{\mathrm{ij}}(X)$ by $K /(N-1)$ and then adding a small self-inhibitory weight $\left(0<-\mathbf{C}_{\mathbf{i i}}<\left|\mathbf{C}_{\mathbf{i j}}\right|\right)$ to each node. A separate constraint optimization was applied to this weight so that the variance of each node, as indicated by the sum of the diagonal terms of $\operatorname{COV}(\mathrm{X})$, was held at a fixed value (0.015). When calculating interactive dynamics, we repeatedly applied a vector $($ size $N$ ) of small scaling factors to the first derivative of the activity matrix $\dot{\mathbf{F}}$ such each node had a variance of 0.015 .

\section{About the Authors}
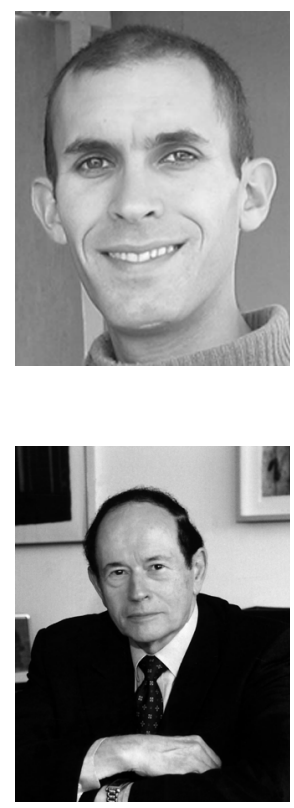

Anil K. Seth obtained a BA (Hons) in Natural Sciences from Cambridge University (1994), where he received the Richards Prize and was elected a scholar of King's College three times. From Sussex University he obtained an MSc with distinction in Knowledge-Based Systems (1996), and a DPhil in Computer Science and Artificial Intelligence (2001). He joined The Neurosciences Institute in 2001 as a Postdoctoral Fellow in Theoretical Neurobiology. His current interests include theoretical neuroanatomy, brain-based neural network modeling, and magnetic brain imaging.

Gerald M. Edelman MD (University of Pennsylvania), PhD (The Rockefeller Institute) is Founder and Director of The Neurosciences Institute (Torrey Pines Mesa, La Jolla, California), a privately supported, not-for-profit center for research focused on understanding the biological basis of higher brain functions in human beings. He is also Professor at the nearby Scripps Research Institute and Chairman of its Department of Neurobiology. He has made many significant research contributions in a variety of fields of biomedical research: his early studies on the structure of antibodies while on the faculty of The Rockefeller University led to his being awarded the Nobel Prize for Physiology for Medicine in 1972; he later moved into the area of brain research where he discovered cell adhesion molecules and made a number of major advances in understanding how nervous systems develop; he has formulated a detailed theory to explain the development and organization of higher brain functions in terms of a process known as neuronal group selection or Neural Darwinism. This theory has been presented in several books, including his newest work, Wider than the sky: The phenomenal gift of consciousness (Yale University Press, 2004), which is being sold internationally. In addition to the Nobel Prize, Professor Edelman has been the recipient of numerous awards and honors, including many honorary degrees. He is a member of the National Academy of Sciences, the American Philosophical Society, and several foreign societies, including the Academy of Sciences, Institute of France. He is the author of more than 450 research publications. Address: The Neurosciences Institute, 10640 John Jay Hopkins Drive, San Diego, CA 92121, USA. E-mail: edelman@nsi.edu 